\title{
Engineering Molecular Ligand Shells on Quantum Dots for Quantitative Harvesting of Triplet Excitons Generated by Singlet Fission
}

Jesse R. Allardice, ${ }^{\dagger}$ Arya Thampi, ${ }^{\dagger}$ Simon Dowland, ${ }^{\dagger}$ James Xiao, ${ }^{\dagger}$ Victor Gray, $^{\dagger, \| \odot ~}$ Zhilong Zhang, $^{\dagger \oplus}$ Peter Budden, ${ }^{\dagger}$ Anthony J. Petty, II, ${ }^{\dagger}$ Nathaniel J. L. K. Davis, ${ }^{\S, \dagger}$ Neil C. Greenham, ${ }^{\dagger}$ John E. Anthony, ${ }^{\ddagger}$ and Akshay Rao* ${ }^{*} \dagger$ (i)

${ }^{\dagger}$ Cavendish Laboratory, University of Cambridge, J. J. Thomson Avenue, Cambridge CB3 OHE, United Kingdom

${ }^{\ddagger}$ Center for Applied Energy Research, University of Kentucky, Research Park Drive, Lexington, Kentucky 40511, United States

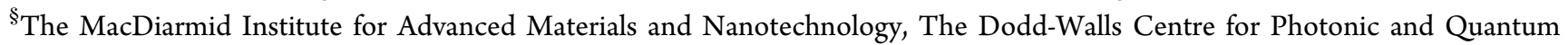
Technologies, School of Chemical and Physical Sciences, Victoria University of Wellington, Wellington 6140, New Zealand

"Department of Chemistry, Ångström Laboratory, Uppsala University, Box 532, Uppsala SE-751 20, Sweden

Supporting Information

ABSTRACT: Singlet fission is an exciton multiplication process in organic molecules in which a photogenerated spin-singlet exciton is rapidly and efficiently converted to two spin-triplet excitons. This process offers a mechanism to break the Shockley-Queisser limit by overcoming the thermalization losses inherent to all single-junction photovoltaics. One of the most promising methods to harness the singlet fission process is via the efficient extraction of the dark triplet excitons into quantum dots (QDs) where they can recombine radiatively, thereby converting high-energy photons to pairs of low-energy photons, which can then be captured in traditional inorganic PVs such as Si. Such a singlet fission photon multiplication (SF-PM) process could increase the efficiency of the best Si cells from $26.7 \%$ to $32.5 \%$, breaking the Shockley-Queisser limit. However, there has been no demonstration of such a singlet fission photon multiplication (SF-PM) process in a bulk system to date. Here, we demonstrate a solution-based bulk SF-PM system based on the singlet fission material TIPS-Tc combined with PbS QDs. Using a range of steady-state and timeresolved measurements combined with analytical modeling we study the dynamics and mechanism of the triplet harvesting process. We show that the system absorbs $>95 \%$ of incident photons within the singlet fission material to form singlet excitons, which then undergo efficient singlet fission in the solution phase $(135 \pm 5 \%)$ before quantitative harvesting of the triplet excitons $(95 \pm 5 \%)$ via a low concentration of QD acceptors, followed by the emission of IR photons. We find that in order to achieve efficient triplet harvesting it is critical to engineer the surface of the QD with a triplet transfer ligand and that bimolecular decay of triplets is potentially a major loss pathway which can be controlled via tuning the concentration of QD acceptors. We demonstrate that the photon multiplication efficiency is maintained up to solar fluence. Our results establish the solution-based SF-PM system as a simple and highly tunable platform to understand the dynamics of a triplet energy transfer process between organic semiconductors and QDs, one that can provide clear design rules for new materials.

\section{INTRODUCTION}

The quest to increase the efficiency of solar energy harvesting has been a major scientific challenge since the invention of the photovoltaic cell (PV). ${ }^{1}$ Single-junction cells made from semiconductors such as silicon and GaAs have been well optimized and attain very high efficiencies of $26.7 \%$ and $29.1 \%$, respectively. ${ }^{2,3}$ However, the efficiency of all single-junction cells is fundamentally capped by the Shockley-Queisser limit. ${ }^{4}$ There is thus a need to develop technologies that can overcome these fundamental limits to the efficiency of single junction cells.
Singlet fission is an exciton multiplication process occurring in a variety of organic semiconductor materials. ${ }^{5,6}$ Here, one photogenerated spin- 0 singlet exciton is converted to two spin1 triplet excitons via a spin-allowed mechanism. Shortly after the discovery of the singlet exciton fission process $(1968)^{7-10}$ it was proposed as a route to break the Shockley-Queisser limit (1979) by reducing the energy lost by thermalization of photoexcited charge carriers with excess energy above the band gap. ${ }^{11}$ However, while there has been a larger effort in recent

Received: June 20, 2019

Published: July 23, 2019 


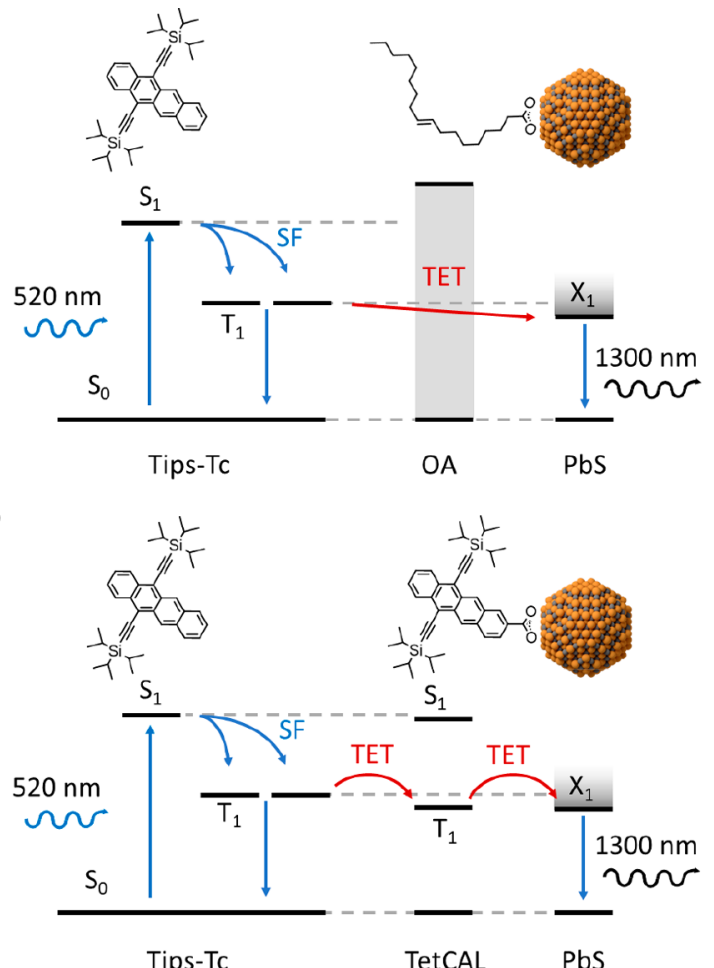

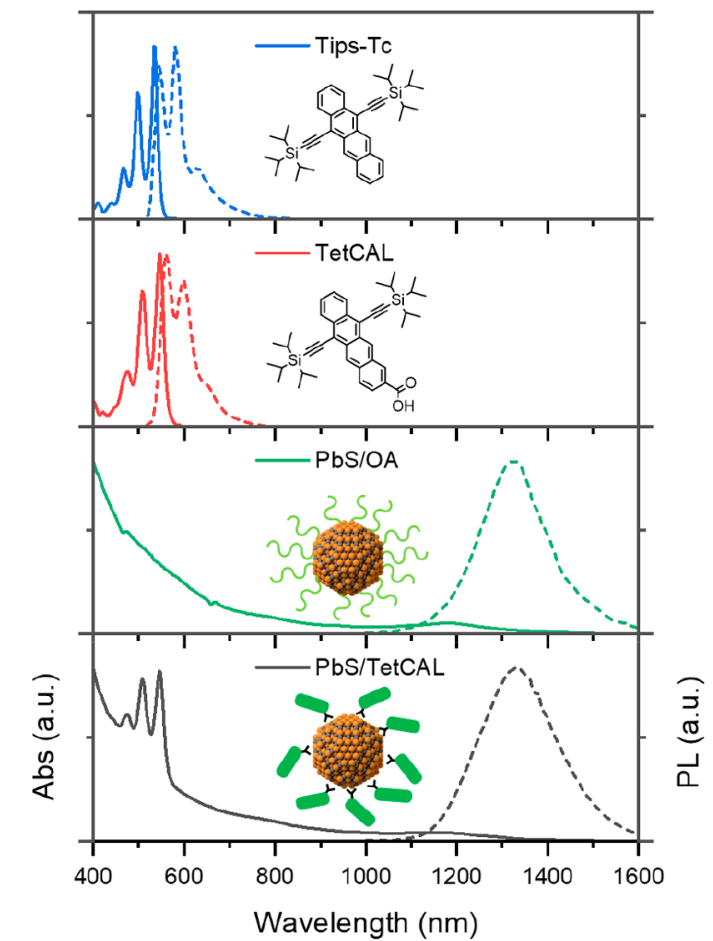

Figure 1. TIPS-Tc, TetCAL, and PbS NCs act as the singlet fission material, transmitter, and emitter, respectively, in this hybrid photon multiplier system. ( $a, b)$ Schematics of the photon multiplication process. TIPS-Tc molecules absorb high-energy visible photons, producing a photoexcited $S_{1}$ state which then interacts with a different ground-state molecule to undergo singlet fission to form two excited triplet molecules via an intermediate TT state. (a) High-band-gap carboxylic acid inhibits the TIPS-Tc triplets from getting close enough to the PbS NCs for triplet energy transfer (TET) to occur. (b) TetCAL molecules bound to the PbS NCs surface are populated via TET from the TIPS-Tc, bringing the triplets in close contact with the PbS NCs and thus mediating TET to the PbS NCs. (c) Absorbance (solid line) and emission (dashed line) spectra of TIPSTc (blue/top), TetCAL (red/top-mid), PbS-OA NCs (green/bottom-mid), and PbS-TetCAL NCs (black/bottom).

years to develop new singlet fission molecules and understand the fundamental photophysics of the process, there have been only a few studies of how to harvest the triplet excitons generated via fission to improve the efficiency of inorganic PV cells, such as Si cells. ${ }^{12-15}$

One of the most promising methods to harness fission is to harvest the energy of the fission-generated triplets via luminescence. ${ }^{16}$ In such a scheme each high-energy photon absorbed by the singlet fission materials would lead to the formation of two triplet excitons via fission which would then be converted to two low-energy photons to be absorbed by a conventional inorganic PV cell, thus doubling the photocurrent from the high-energy part of the solar spectrum. This scheme, termed a singlet fission photon multiplier (SF-PM), has been described recently and its potential effect on cell efficiencies calculated. ${ }^{17}$ It was shown that it could increase the efficiency of the best Si PV cells available today from $26.7 \%$ to $32.5 \%$, thus breaking through the Shockley-Queisser limit for the silicon band gap. The SF-PM is also technologically attractive as it does not require modification of the underlaying inorganic PV but rather can be coated on top of it.

Since triplet excitons are dark states, due to their spinforbidden return to the ground state, they cannot directly emit photons. ${ }^{5}$ Hence, a key breakthrough was the demonstration of transfer of triplet excitons to colloidal inorganic quantum dots (QDs), where the excitations become bright and can recombine to emit photons. ${ }^{18,19}$ This discovery also led to the study of the reverse process, the transfer of energy from QDs to the triplet state of organic semiconductors, for application in upconversion and triplet sensitization to drive photochemical reactions. ${ }^{20,21}$ Numerous studies in this area have focused on the role of the ligand on the QD in facilitating or hindering transfer of energy to the organic semiconductor. $^{22-24}$ These ligands both passivate surface defects and provide the QDs with colloidal stability. The transfer dependence on the length of the ligands indicated a Dexter-like transfer mechanism with shorter ligands providing more efficient transfer as the ligands serve as a tunnel barrier. ${ }^{25,26}$ However, for the transfer of triplets into QDs, the basis of current SF-PM technologies, there have been no equivalent studies looking at triplet harvesting in bulk systems. The two previous reports of triplet transfer to QDs considered bilayer systems containing layers of organic and QDs on top of each other. $^{18,19}$ The confined and bilayer nature of the system means that triplets formed via fission are always close to an interface with the QDs and hence have ample opportunity to tunnel across the ligands. However, such a scheme does not provide sufficient light absorption as to be of any practical use. In a useful SF-PM, the singlet fission material must be present in sufficient quantity to harvest most of the incident photons (>95\%) and at the same time the QDs must be present in a low concentration so as to minimize parasitic loss via absorption of solar photons by QDs $(<5 \%) .{ }^{17}$ No such bulk system has yet been demonstrated, and the dynamics of the triplet transfer process to the QDs in such system remain unexplored.

Here, we report a solution-based bulk SF-PM system in which $>95 \%$ of incident photons are absorbed by the singlet 

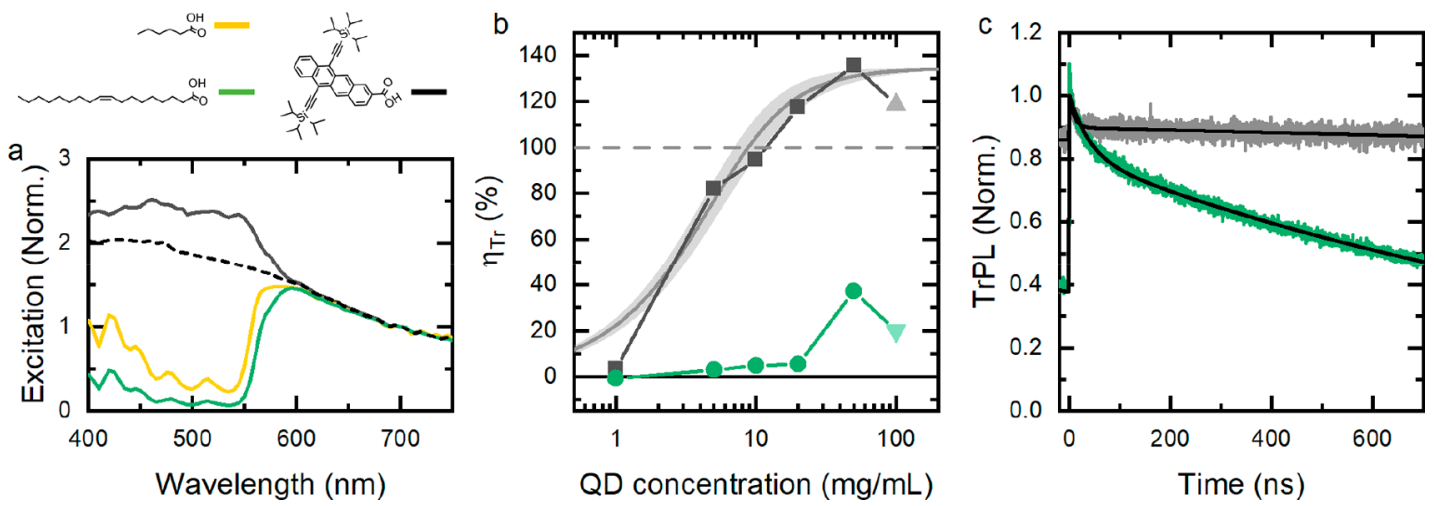

Figure 2. (a) PbS QD PL excitation spectra for solutions of TIPS-Tc $(200 \mathrm{mg} / \mathrm{mL})$ and $1.05 \mathrm{eV} \mathrm{PbS} \mathrm{QDs}(50 \mathrm{mg} / \mathrm{mL})$ with OA (green), HA (yellow), and TetCAL (gray) ligands along with PbS/OA QDs on their own (dashed). Excitation spectra are normalized to the value at $700 \mathrm{~nm}$, where only the $\mathrm{QD}$ is absorbing. (b) Exciton transfer efficiencies from TIPS-Tc to PbS/OA (green circles) and PbS/TetCAL (black squares) in solution with varying concentrations together with TIPS-Tc $(200 \mathrm{mg} / \mathrm{mL})$ under $515 \mathrm{~nm} 500 \mu \mathrm{W} / \mathrm{cm}^{2}$ excitation. Horizontal gray dashed line indicates the point at which $100 \%$ exciton transfer occurs. Values for $100 \mathrm{mg} / \mathrm{mL}$ QD concentration have been highlighted as outliers due to selfabsorption losses. Triplet exciton transfer efficiency, $\eta_{\text {TET }}$, calculated with the kinetic parameters derived from the nsTA (details below) is scaled by a singlet fission efficiency, $\eta_{\mathrm{SF}}=1.35 \pm 0.05$, to match with the values obtained by PLQE measurements for PbS/TetCAL (gray, with $95 \%$ confidence bounds). (c) Near-infrared transient PL for $10 \mathrm{mg} / \mathrm{mL}$ PbS/OA NCs (green) and PbS/TetCAL NCs (gray) in toluene with $100 \mathrm{mg} /$ $\mathrm{mL}$ TIPS-Tc, under excitation with $530 \mathrm{~nm} 300 \mathrm{pJ} / \mathrm{cm}^{2}, 1 \mathrm{MHz}$ repetetion rate pump pulses. The kinetics have been normalized to the maximum value after removal of a fixed value representative of contributions to camera counts from ambient conditions. Laser pump timing has been aligned with $t=0 \mathrm{~ns}$ and thus counts before this time are residual counts from all previous pump pulses. Fits to the transient kinetics (black) follow a parametrization with a biexponential function where the slower exponential decay is summed over all previous pump pulses, representing an exponential decay in a periodic driven system (see supplementary section 7 for details).

fission material. Efficient singlet fission then occurs in the solution phase $(135 \pm 5 \%)$ before the triplet excitons are quantitatively $(95 \pm 5 \%)$ harvested via a low concentration of QDs $(<50 \mathrm{mg} / \mathrm{mL})$, followed by emission of IR photons. Our solution-phase SF-PM consists of a blend of a highly soluble singlet fission material, 5,12-bis((triisopropylsilyl)ethynyl)tetracene) referred to as TIPS-Tc, ${ }^{27}$ and lead sulfide (PbS) QDs covered in 6,11-bis((triisopropylsilyl)ethynyl)tetracene2-carboxylic acid)) ligands, referred to as TetCAL, together in toluene solution. The TIPS-Tc absorbs photons via its $S_{0} \rightarrow S_{1}$ transition $(2.32 \mathrm{eV})$, while the triplets generated by singlet fission are efficiently transferred to the $\mathrm{PbS} \mathrm{QDs}$ via the TetCAL ligands, resulting in IR luminescence (Figure 1b). Using a range of steady-state and time-resolved measurements combined with analytical modeling we study the dynamics and mechanism of the triplet harvesting process and show that in order to achieve efficient triplet harvesting it is critical to engineer the surface of the QD with a triplet transfer ligand, TetCAL, analogous to recent work with QD-organic based upconversion systems. ${ }^{23}$ Our results demonstrate that under solar-equivalent fluences it is possible to efficiently harvest triplet excitons in a bulk system via a low concentration of QDs with sufficiently low QD parasitic absorption for realistic coupling to a Si PV cell and establish design rules for such a process. ${ }^{17}$ Our solution-based system also serves as a simple and highly tunable platform to understand the dynamics of the triplet energy transfer process between organic semiconductors and QDs.

\section{RESULTS AND DISCUSSION}

Characterization of TIPS-Tc+PbS/TetCAL Solution SFPM System. The synthesis of PbS QDs with oleic acid ligands (OA) and subsequent ligand exchange with either TetCAL or hexanoic acid (HA) was carried out using an adaptation of previously reported methods. ${ }^{28}$ Figure 1c shows the absorbance and emission spectra of TIPS-Tc, TetCAL, and
$\mathrm{PbS}$ QDs with the native OA ligands ( $\mathrm{PbS} / \mathrm{OA}$ ) and those modified with TetCAL via ligand exchange (PbS/TetCAL). Attachment of the TetCAL ligand after multiple wash cycles in acetone is confirmed via UV-Vis absorption measurements, where the TetCAL absorbance peaks are visible on top of the $\mathrm{PbS}$ QD absorbance. The absorbance of TIPS-Tc, TetCAL, and $\mathrm{PbS} / \mathrm{TetCAL}$ show clear vibrionic structure. The $0-0$ vibrionic peak of TIPS-Tc at $535 \mathrm{~nm}$ gives an $S_{1}$ energy of 2.32 $\mathrm{eV}$, while the TetCAL $0-0$ peak at $545 \mathrm{~nm}(2.28 \mathrm{eV})$ indicates a $40 \mathrm{meV}$ red shift on addition of the carboxylic acid functional group. The triplet energy of TIPS-Tc is expected to be 1.2 $\mathrm{eV}^{27}$ meaning that singlet fission in TIPS-Tc is endothermic.

The $\mathrm{PbS}$ QDs are tuned such that their band gap, as measured from the excitonic absorption peak at $\sim 1180 \mathrm{~nm}$ $(\sim 1.05 \mathrm{eV})$, is below the triplet energy of TIPS-Tc $(\sim 1.2 \mathrm{eV})$, making it energetically favorable to accept triplets from TIPSTc. ${ }^{18}$ The Stokes-shifted PbS QD photoluminescence peak is at $\sim 1350 \mathrm{~nm}(\sim 0.92 \mathrm{eV})$. TIPS-Tc is a well-studied singlet fission material, which has been shown to efficiently undergo fission in high-concentration solutions $(>200 \mathrm{mg} / \mathrm{mL})$ with a fission yield of $120 \pm 10 \% .^{27,29}$

TetCAL is designed to act as a triplet transmitter ligand, whose triplet energy will lie above the band gap of the QDs and slightly below that of the TIPS-Tc fission material, due to the conjugation of the $\mathrm{COOH}$ group which slightly lowers the energy levels in comparison to TIPS-Tc. As illustrated in Figure 1a, transfer of a triplet between TIPS-Tc and the $\mathrm{PbS} /$ $\mathrm{OA} \mathrm{QD}$ would have to occur over a large distance. The oleic acid ligands act as a tunnelling barrier, resulting in a large Dexter transfer distance and thus reducing the rate of transfer, $^{18,19}$ whereas with the TetCAL ligand acting as a transmitter, the triplet exciton can first transfer to the ligand. After this initial triplet transfer the Dexter transfer distance into the $\mathrm{PbS} \mathrm{QD}$ has been significantly decreased compared to transfer through either OA or HA. ${ }^{23}$ 
Molar attenuation coefficients for the various species were measured across the vis-NIR range. In particular, the molar attenuation coefficients at $515 \mathrm{~nm}$ for TIPS-Tc, PbS/OA, and $\mathrm{PbS} /$ TetCAL were found to be $2.4 \times 10^{4}, 2.6 \times 10^{5}$, and $3.5 \times$ $10^{5} \mathrm{~L} \mathrm{~mol}^{-1} \mathrm{~cm}^{-1}$, respectively (Figure S2). These attenuation coefficients have been used to calculate the relative absorption of photons in each species for varying concentrations of the blend components. The measured absorbance of a $15 \mu \mathrm{m}$ thick solution of TIPS-Tc and PbS/TetCAL QDs shows the TIPSTc absorbance peak $(535 \mathrm{~nm})$ is 2 orders of magnitude higher than the parasitic absorbance of the $\mathrm{PbS} \mathrm{QD}$ (Figure S4a). Using the molar attenuation coefficients for the solution PM the absorption for a $2.5 \mu \mathrm{m}$ thick solution is predicted to be $>95 \%$ at the TIPS-Tc peak, while the QD parasitic absorption will be less than $5 \%$, thus fulfilling the absorption criteria for a "realistic" SF-PM proposed previously (Figures S4c and S4d). ${ }^{17}$

We perform qualitative evaluation of the SF-PM system by measuring IR-detected photoluminescence excitation spectra. Figure 2a shows the photoluminescence (PL) excitation scan of a solution of $\mathrm{PbS} / \mathrm{OA} \mathrm{QDs}$ in toluene $(50 \mathrm{mg} / \mathrm{mL})$ along with the comparable excitation scans for blends of TIPS-Tc $(200 \mathrm{mg} / \mathrm{mL})$ and QDs with various ligands, normalized to the value at $700 \mathrm{~nm}$ excitation. ${ }^{18}$ The excitation scan of $\mathrm{PbS} / \mathrm{OA}$ has a decreasing emission with increasing wavelength, following the absorbance of the QDs across this region. At wavelengths above $600 \mathrm{~nm}$, where only the QDs are absorbing, all solutions follow the same trend. However, at wavelengths less than this, the concentrated TIPS-Tc with orders of magnitude higher absorbance, is absorbing most of the light (Figure S4), and so the IR PL from the solution is an indication of the amount of exciton transfer from TIPS-Tc to the QDs. Compared to PbS/OA on its own, TIPS-Tc+PbS/ TetCAL solution shows an increase in the IR PL for wavelengths where the TIPS-Tc is absorbing, with the PL excitation peaks matching with TIPS-Tc absorption peaks, indicating a high exciton transfer efficiency. In contrast, blends of TIPS-Tc and PbS QDs without the TetCAL ligand (either OA or HA ligands) show a significant drop in IR PL for excitation below $550 \mathrm{~nm}$ with dips that match with the absorption peaks of TIPS-Tc. This shows that for these solutions energy transfer from TIPS-Tc to the QDs is inefficient.

For quantitative evaluation of the SF-PM system we use IR PLQE measurements on a series of solutions with varying $Q D$ concentrations. By comparing the IR PLQE values when the solution is excited at $515 \mathrm{~nm}$, which excites both TIPS-Tc and $\mathrm{QD}$, or at $658 \mathrm{~nm}$, which selectively excites the QDs, the efficiency of exciton transfer can be determined (Figure $2 b$ ). For TIPS-Tc+PbS/TetCAL solutions the peak PLQE occurs at a QD concentration of $50 \mathrm{mg} / \mathrm{mL}$, with $18.2 \%$ IR PLQE ( 515 $\mathrm{nm}$ excitation), while the intrinsic PLQE of the QD in the same solution was found to be $14.6 \%$ (658 nm excitation) (Table S1).

The IR PLQE of a photon multiplier, $\eta_{\mathrm{PM}}(\lambda)$, for excitation at wavelength $\lambda$, with singlet fission donor and emissive QD acceptor components can be expressed as ${ }^{18}$

$$
\eta_{\mathrm{PM}}(\lambda)=\eta_{\mathrm{QD}} \frac{\mu_{\mathrm{QD}}(\lambda)+\eta_{\mathrm{Tr}} \mu_{\mathrm{Tc}}(\lambda)}{\mu_{\mathrm{QD}}(\lambda)+\mu_{\mathrm{Tc}}(\lambda)}
$$

where $\eta_{\mathrm{QD}}$ is the intrinsic PLQE of the QD, $\mu_{i}$ is the attenuation coefficient, base 10 , of the $i$ th component, and $\eta_{\operatorname{Tr}}$ is the total exciton transfer efficiency from the donor to acceptor. Using eq 1 , the measured molar absorption coefficients, and the intrinsic QD PLQEs we calculate the exciton transfer efficiency, $\eta_{\operatorname{Tr}}$, as shown in Figure $2 \mathrm{~b}$ (see supplementary section 4 for details). Here, we have quantitative proof of singlet fission photon multiplication, as we observe values of exciton transfer above $100 \%$, for concentrations greater than $\sim 10 \mathrm{mg} / \mathrm{mL}$ of $\mathrm{PbS} / \mathrm{TetCAL}$ QDs. Magnetic field-dependent PL measurements confirm that we are harvesting triplet excitons generated via singlet fission (Figure S31). ${ }^{18}$

The PLQE and transfer efficiency values for $100 \mathrm{mg} / \mathrm{mL}$ QD concentration have been highlighted as outliers due to selfabsorption losses (Figure 2b). Self-absorption is identified from the drop in intrinsic PLQE and red shifting of the PL spectrum (Figure S6). The measured transfer efficiencies, $\eta_{\mathrm{Tr}}$, for the $\mathrm{PbS} / \mathrm{OA}$ system are low for all $\mathrm{QD}$ concentrations, indicating poor exciton transfer. Changing the $\mathrm{QD}$ ligand to HA does result in slightly increased IR PL when the TIPS-Tc is absorbing. This trend agrees with the HA ligand resulting in higher exciton transfer than the longer OA ligand due to HA having a shorter Dexter transfer distance. ${ }^{18,19,25}$ However, the TetCAL ligand greatly outperforms the shorter HA ligands.

Along with the increased steady-state PLQE we measure a longer lived transient PL signal for PbS/TetCA+TIPS-Tc compared to $\mathrm{PbS} / \mathrm{OA}+\mathrm{TIPS}-\mathrm{Tc}$ when excited at $530 \mathrm{~nm}$, as shown in Figure $2 \mathrm{c}$. This indicates that the triplet exciton transfer is occurring on time scales comparable to or slower than the decay of the excited QD states. Due to the long lifetime of the QDs compared to the fixed repetition rate (1 $\mathrm{MHz}$ ) used to photoexcite the system, a significant population of excited TIPS-Tc and QD states were still present when the next pump laser pulse interacted with the system. This effect is most apparent by the high PL counts before time zero. This represents real photon counts from the sample, as the electronic noise that contributes a background has been removed (supplementary section 7 ). We use a biexponential decay in the presence of a periodic excitation to fit the decay of the QD PL (supplementary section 7). The short time constant component is a parametrization of the nonlinear recombination occurring in the $\mathrm{QD}$, while the longer time constant is the decay constant for excited QD states. When the SF-PM solutions are excited at $530 \mathrm{~nm}$, where TIPS-Tc's absorption is dominant, we extract a decay constant of $1.30 \pm$ $0.01 \mu$ s for the $\mathrm{PbS} / \mathrm{OA}$ quantum dots compared to $22.0 \pm 0.7$ $\mu$ s for the PbS/TetCAL QDs. The value for PbS/OA QDs is in agreement with previous reports for $\mathrm{PbS} / \mathrm{OA} \mathrm{QDs}$ alone in toluene; however, the $\mathrm{PbS} / \mathrm{TetCAL}$ value is significantly longer. $^{22}$ This suggests the TIPS-Tc triplet excited states are feeding the PbS/TetCAL QDs $(10 \mathrm{mg} / \mathrm{mL})$ with a time constant of around $20 \mu \mathrm{s}$.

Investigation of TIPS-Tc Singlet Fission by Femtosecond Transient Absorption. To evaluate the ultrafast excited-sate dynamics of TIPS-Tc in the presence of PbS QDs, femtosecond transient absorption spectra were measured (Figures S10 and S11). In concentrated solutions of TIPSTc $(200 \mathrm{mg} / \mathrm{mL})$, with and without PbS/TetCAL (50 mg/ $\mathrm{mL}$ ), we observe a loss of the singlet and rise of triplet features within 100 ps after excitation (supplementary section 8). ${ }^{27,29}$ Comparing the decay of the singlet exciton in both cases reveals no significant difference in the fission kinetics and shows that singlet exciton transfer to the QD cannot compete with the singlet fission rate. We put an upper bound on singlet 
a

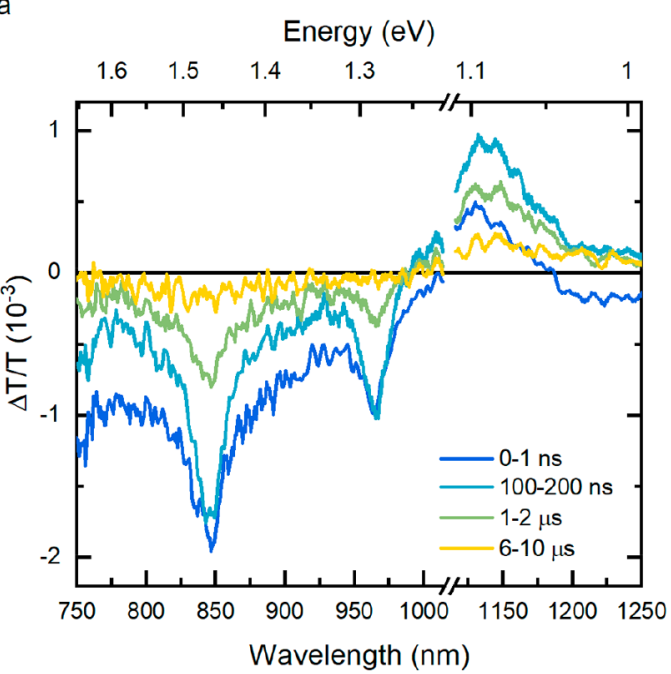

C

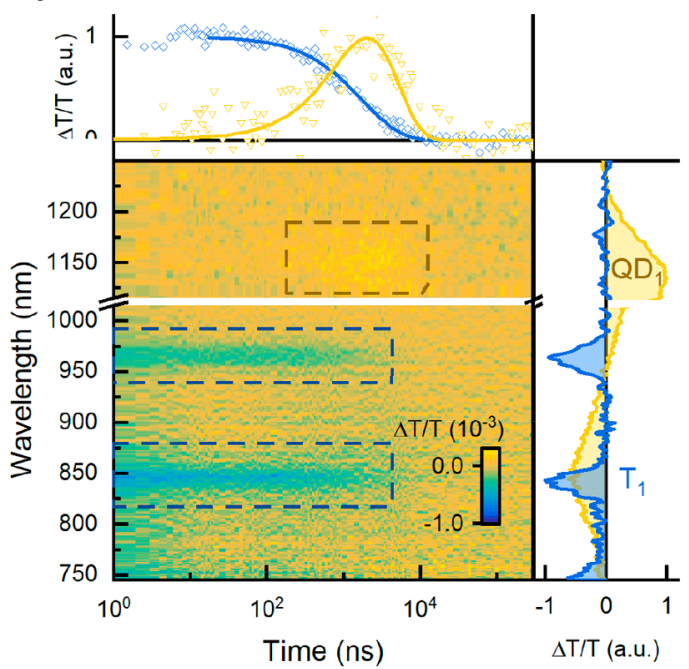

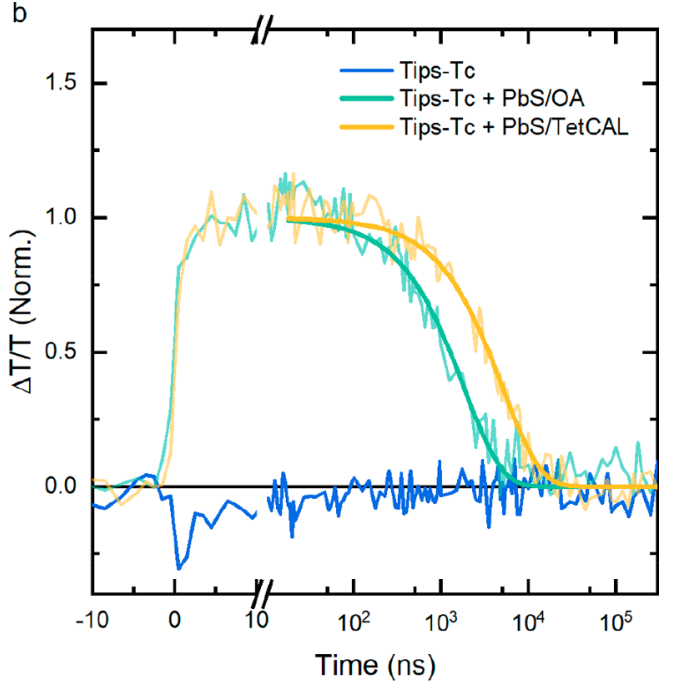

d

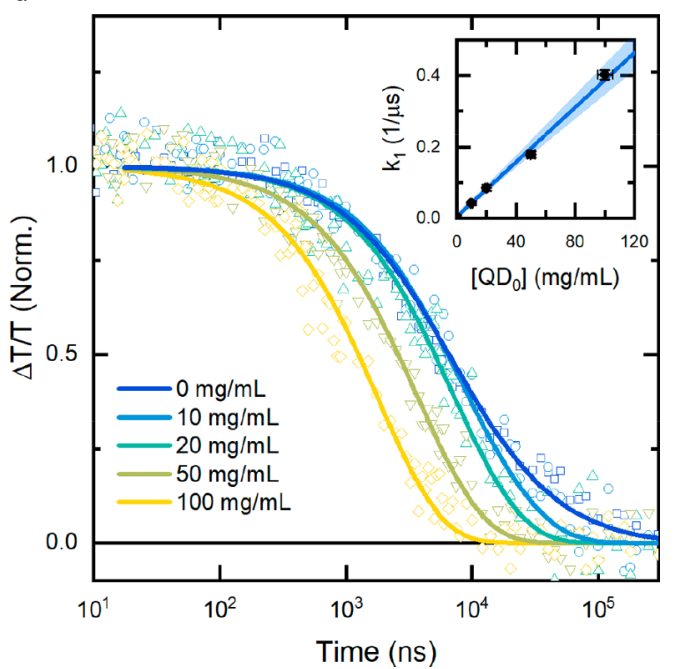

Figure 3. (a) nsTA spectra of TIPS-Tc+PbS/TetCAL blend $(200$ and $100 \mathrm{mg} / \mathrm{mL})$ excited at $532 \mathrm{~nm} 170 \mu \mathrm{J} / \mathrm{cm}^{2}$. Each spectrum is an average over the time ranges indicated. (b) Normalized nsTA kinetics under $40 \mu \mathrm{J} / \mathrm{cm}^{2}$ at the QD GSB region $(1140-1160 \mathrm{~nm})$. QD GSB signals have been fitted with a monoexponential decay. $\mathrm{PbS} / \mathrm{OA}$ and $\mathrm{PbS} / \mathrm{TetCAL} \mathrm{QDs}$ are found to decay with a $1.8 \pm 0.1$ and $5.1 \pm 0.2 \mu \mathrm{s}$ decay constant, respectively; $1140-1160 \mathrm{~nm}$ kinetic for TIPS-Tc has been scaled by the same normalization factor as for PbS/TetCAL+TIPS-Tc. (c) nsTA difference map for a solution of TIPS-Tc $(200 \mathrm{mg} / \mathrm{mL})$ and PbS/TetCAL QDs $(100 \mathrm{mg} / \mathrm{mL})$ under $535 \mathrm{~nm} 40 \mu \mathrm{J} / \mathrm{cm}^{2}$ excitation. PbS/TetCAL and TIPS-Tc triplet TA spectra used for decomposition by linear regression are shown (right inset). Strengths of TIPS-Tc triplet PIA signal (from linear regression) and $\mathrm{PbS} /$ TetCAL QD GSB $(1140-1160 \mathrm{~nm}$ ) are shown with overlaid simulation of the population (top inset). (d) Normalized kinetics for the TIPS-Tc triplet population found from decomposition via linear regression of the corresponding nsTA difference map for a variety of $\mathrm{PbS} / \mathrm{TetCAL} \mathrm{QD}$ concentrations. Triplet decay kinetics were fitted with an analytical solution for a second-order rate equation, where the bimolecular triplet decay rate is the value found for pristine TIPS-Tc. (Inset) Fitted monomolecular triplet decay rate constant as a function of $\mathrm{PbS} /$ TetCAL QD concentration with a linear fit and $95 \%$ confidence bounds.

exciton transfer efficiency from the initially excited TIPS-Tc singlet to the $\mathrm{PbS} / \mathrm{TetCAL}$ QDs at $5 \%$ (supplementary section 8). We see no significant growth of QD features in the first 2 ns after photoexcitation. Therefore, the transfer being observed is predominantly triplet transfer on time scales greater than 2 ns.

Investigation of Triplet Transfer by Nanosecond Transient Absorption. To investigate the full decay of the excited states in TIPS-Tc and PbS/TetCAL, nanosecond transient absorption (nsTA) spectra were measured, as shown in Figure 3a. After femtosecond pulse excitation at $535 \mathrm{~nm}$ we observe initial nsTA spectra that contain both TIPS-Tc triplet and excited-state QD features in the NIR probe region (750$1250 \mathrm{~nm}$ ). We identify the TIPS-Tc triplet excitons by the two photoinduced absorption (PIA) peaks at $840-850$ and 960-
$970 \mathrm{~nm} .^{27,29}$ The positive signal at $950-1200 \mathrm{~nm}$ is assigned to a ground-state bleach (GSB) from QD excited states. ${ }^{19}$

In solutions of concentrated pristine TIPS-Tc we observe long-lived $(>10 \mu \mathrm{s})$ triplet excitons as identified by the triplet PIA features (Figure S12), which is consistent with previous literature. $^{27}$ Additionally, we observe a broad PIA feature across the probe range, identified as an excimer state, decaying within $10 \mathrm{~ns}^{27,29}$ The decay of TIPS-Tc triplets display significant fluence dependence, indicating bimolecular recombination as a significant decay channel for the excited triplet states (Figure S12). Global fitting of multiple nsTA kinetics (at varying laser fluences), following previously reported methods for fitting triplet decay dynamics, involving an analytic model for a second-order rate equation, allows extraction of the monomolecular and bimolecular triplet decay rates of $5.6 \pm 5.1$ 

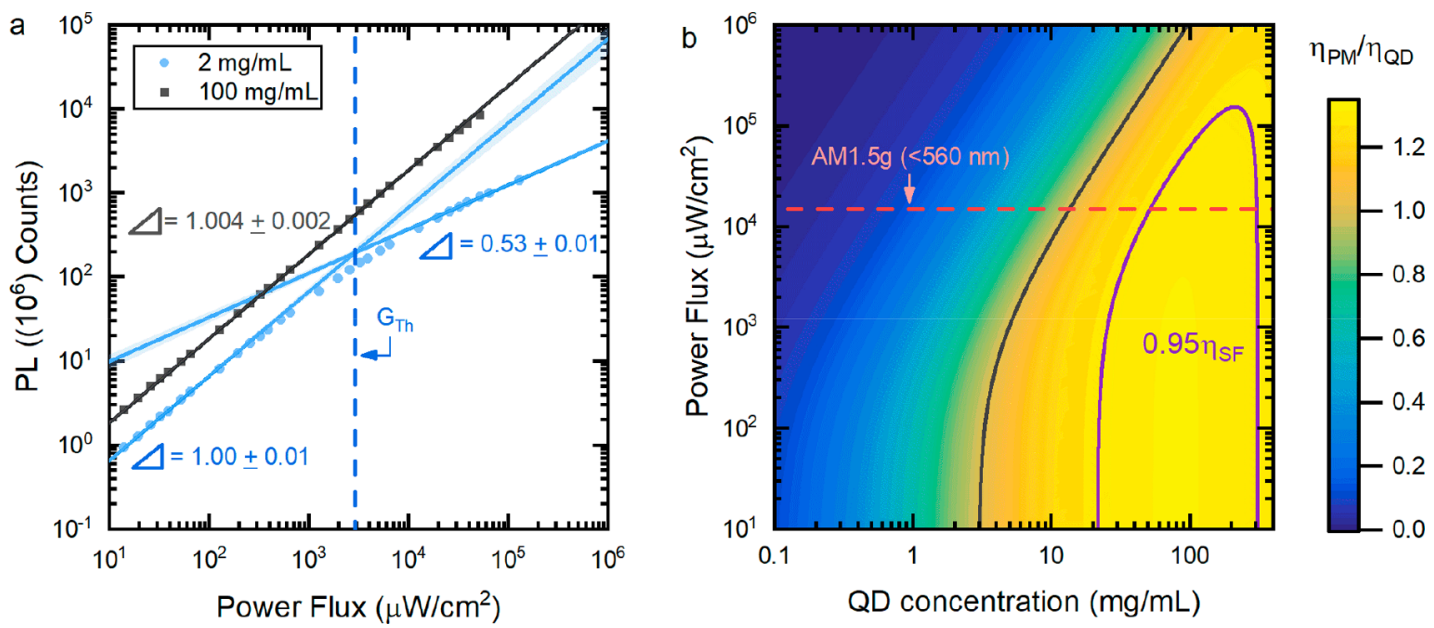

Figure 4. (a) Total IR PL counts from PbS/TetCAL QDs for solutions of low ( $2 \mathrm{mg} / \mathrm{mL}$, blue circles) and high (100 mg/mL, black squares) QD concentration with TIPS-Tc $(200 \mathrm{mg} / \mathrm{mL})$ for varying $532 \mathrm{~nm}$ excitation flux. PL counts are fit with power-law relations to laser flux either across the entire flux range $(100 \mathrm{mg} / \mathrm{mL})$ or separated into two fits $(2 \mathrm{mg} / \mathrm{mL})$ for low and high photon flux. Intercept of the fits to the low- and high-flux regimes gives $2.9 \pm 1.0 \mathrm{~mW} / \mathrm{cm}^{2}$ as the threshold power flux (blue vertical dashed line). (b) Simulation of the photon multiplication efficiency normalized by the PbS/TetCAL QD intrinsic PLQE under $532 \mathrm{~nm}$ excitation. Two contours of interest are highlighted: region in which the PM efficiency is larger than the QD PL efficiency $\eta_{\mathrm{QD}}$ (black) and when PM efficiency is $95 \%$ of the upper limit for PM efficiency given by the singlet fission yield (purple line). Equivalent solar flux available for absorption by TIPS-Tc under the AM1.5G spectrum (red horizontal dashed line).

$\mathrm{ms}^{-1}$ and $(7.6 \pm 0.3) \times 10^{-23} \mathrm{~cm}^{3} \mathrm{~ns}^{-1}$, respectively (Table S5). ${ }^{30}$ The significant uncertainty on the monomolecular decay rate indicates that we have not fully resolved the intrinsic decay of the triplet states. Given the uncertainty in the extracted values, comparison of the decay rate shows at most $40 \%$ of triplets decay monomolecularly at the lowest laser fluence used, $21 \mu \mathrm{J} / \mathrm{cm}^{2}$, the rest decaying via bimolecular channels.

In the presence of either $\mathrm{PbS} / \mathrm{OA}$ or $\mathrm{PbS} / \mathrm{TetCAL}$ we observe no effect on the generation of triplets via singlet fission in TIPS-Tc, evident by the similar initial nsTA intensity of the triplet PIA features (Figure S16). For both QDs types, at early times the negative nsTA feature corresponding to the TIPS-Tc excimer PIA overlaps with the GSB and PIA of the QDs. The decay of this negative feature, produces an apparent rise in the positive ground-state QD GSB signals which overlap in the $1140-1160 \mathrm{~nm}$ region. This rise in signal is thus not associated with a change in $\mathrm{QD}$ population (Figure $3 \mathrm{~b}$ ). At $10 \mathrm{~ns}$, the $\mathrm{PbS} / \mathrm{OA}$ and $\mathrm{PbS} / \mathrm{TetCAL} \mathrm{GSB}$ signals are of similar intensities, indicating similar initial populations of excited QDs in both systems. We assign this initial TA signal to the fraction of photons that directly excite the QDs with the 535 $\mathrm{nm}$ pump pulse. After the initial direct excitation of the $\mathrm{PbS} /$ OA QDs we observe a decay in the excited QD signal characterized by a $1.8 \pm 0.1 \mu$ s decay constant. In comparison the solution with $\mathrm{PbS} / \mathrm{TetCAL} \mathrm{QDs}(100 \mathrm{mg} / \mathrm{mL})$ shows a significantly longer $5.1 \pm 0.2 \mu \mathrm{s}$ decay constant for the QDs. This is longer than its intrinsic $1.90 \pm 0.05 \mu$ s lifetime (Figure S13). This increased time constant is consistent with delayed triplet transfer to the QDs and is thus consistent with the TrPL data shown in Figure 2c.

To clarify the quenching of the TIPS-Tc triplets and transfer to the PbS/TetCAL QDs, difference nsTA maps were calculated. The nsTA spectra for PbS/TetCAL (under 535 $\mathrm{nm}$ excitation) were linearly scaled to match with the initial QD GSB (in the range 20-40 ns) in the TIPS-Tc+PbS/ TetCAL blends, and the difference was calculated. ${ }^{31}$ The nsTA difference maps contain information about excited QDs resulting from transfer without contribution from directly excited $\mathrm{PbS} /$ TetCAL QDs (Figures $3 \mathrm{c}$ and S18). After this transformation the loss of the TIPS-Tc triplets corresponding with a rise in the QD GSB is clear. The strength of the QD GSB that grows in after $100 \mathrm{~ns}$ is seen to increase with the concentration of the QDs (Figure S19), consistent with diffusion-limited transfer. Applying linear regression of the pristine TIPS-Tc triplet spectrum to the difference maps allows extraction of the magnitude of the TIPS-Tc triplet PIA spectrum as a function of time and QD concentration, as seen in Figure 3d (details in section S8). Comparing these triplet PIA lifetimes shows a quenching that is dependent on $\mathrm{PbS} /$ TetCAL QD concentration.

Kinetic Model. We use the following kinetic model to describe the transfer of triplets from the TIPS-Tc to the $\mathrm{PbS} /$ TetCAL QDs

$$
\begin{aligned}
& \frac{\mathrm{d} T_{1}}{\mathrm{~d} t}=-\left(k_{\mathrm{T}}+k_{\mathrm{TET}} X_{0}\right) T_{1}-k_{2} T_{1}^{2} \\
& \frac{\mathrm{d} X_{1}}{\mathrm{~d} t}=k_{\mathrm{TET}} X_{0} T_{1}-k_{X} X_{1}
\end{aligned}
$$

where $T_{1}$ is the triplet density produced via singlet fission with an efficiency $\eta_{\mathrm{SF}}, X_{1}$ is the density of excited QD states, $k_{\mathrm{T}}$ and $k_{2}$ are the intrinsic and bimolecular decay rate constants for TIPS-Tc triplets, $k_{\mathrm{TET}}$ is the triplet transfer rate to the $\mathrm{PbS} /$ TetCAL QDs, $k_{X}$ is the QD decay rate, and $X_{0}$ is the concentration of the $\mathrm{QD}$ acceptor. Using the bimolecular triplet decay rate obtained for pristine TIPS-Tc as a fixed input parameter we apply fitting of an analytical solution for the second-order rate equation and extract the monomolecular decay rate as a function of $\mathrm{PbS} /$ TetCAL QD concentration, $k_{1}$ $=k_{\mathrm{r}}+k_{\mathrm{TET}} X_{0}$ (inset, Figure 3d). Applying this Stern-Volmerlike quenching model we extract a triplet transfer rate of $k_{\mathrm{TET}}=$ $0.0039 \pm 0.0001(\mathrm{mg} / \mathrm{mL})^{-1} \mu \mathrm{s}^{-1}\left((5.1 \pm 0.1) \times 10^{8} \mathrm{M}^{-1} \mathrm{~s}^{-1}\right)$ and an intrinsic triplet lifetime of $\tau_{\mathrm{T}}=250 \pm 180 \mu \mathrm{s} .{ }^{30}$ The reasonable agreement with a linear relation between TET rate and QD acceptor concentration indicates that the triplet transfer is diffusion limited, not kinetically limited. Using triplet exciton diffusion constants from previously reported 
diffusion-ordered nuclear magnetic spectroscopy measurements and assuming relatively low diffusion of the $\mathrm{PbS} /$ TetCAL QD we estimate the diffusion-limited transfer rate to be $0.085 \pm 0.012(\mathrm{mg} / \mathrm{mL})^{-1} \mu \mathrm{s}^{-1}$ (supplementary section 6). ${ }^{27}$ This is roughly a factor of 20 times higher than what we observe. Possible reasons for this disagreement include a low Gibbs free energy to drive the transfer, nonuniform coverage of the TetCAL ligand over the surface of the PbS/TetCAL QDs, and steric hindrance from residual OA ligands attached to the QD. ${ }^{32}$

Using the extracted intrinsic PbS/TetCAL QD lifetime and triplet transfer rate the simulated dynamics for the decay of the triplet excitons and the excited-state QDs are calculated and shown in Figure $3 \mathrm{c}$ along with the TIPS-Tc triplet difference PIA signal and the QD GSB signal (1140-1160 nm) (details in supplementary section 8 ). Agreement with the two species model described by eqs 2 and 3 suggests that the transfer of triplet excitons from the TetCAL molecules into the QD is not rate limiting. However, this step should still be seen as critically important for the transfer process. Additionally, we calculate the triplet transfer efficiency, $\eta_{\mathrm{TET}}$, as a function of the $\mathrm{PbS}$ / TetCAL QD concentration at the laser fluence used in the IR PLQE measurements. Multiplication of this TET efficiency with a singlet fission yield of $\eta_{\mathrm{SF}}=135 \pm 5 \%$ gives reasonable agreement with the observed values for the exciton transfer efficiency $\eta_{\mathrm{Tr}}=\eta_{\text {TET }}$ from our PLQE data (Figure $2 \mathrm{~b}$, gray curve). This value for the singlet fission yield agrees with previous predictions obtained via the magnitude of transient absorption spectra. ${ }^{27}$ For the $50 \mathrm{mg} / \mathrm{mL}$ solution of $\mathrm{PbS} /$ TetCAL QDs we calculate a triplet exciton transfer efficiency of $\eta_{\mathrm{TET}}=95 \pm 5 \%$, approaching $100 \%$ at higher $\mathrm{QD}$ concentration (Figure S27).

Unexpectedly, the nsTA data suggest that triplet exciton transfer in TIPS-Tc+PbS/TetCAL solutions is significantly hindered by bimolecular decay of the TIPS-Tc triplets, presenting a concern for the versatility of this SF-PM to operate in real-world conditions under solar irradiance.

Determining the Solution Phase SF-PM Fluence Dependence. To evaluate the effect of bimolecular triplet decay on the photon multiplication efficiency of TIPS-Tc $+\mathrm{PbS} /$ TetCAL blends, steady-state PL spectra were measured at a range of laser fluences, as shown in Figure 4a. Here we measure the PL from the QDs that arises after fission and triplet transfer. At lower excitation densities the QD IR PL increases linearly with the flux but then passes through a threshold triplet generation density, $G_{\mathrm{Th}}$, after which the QD $\mathrm{PL}$ goes as the square root of the excitation density. Modeling of the system (see supplementary section 10) reveals that the threshold triplet generation rate per unit volume, $G_{\mathrm{Th}}$, is given by

$$
G_{\mathrm{Th}}=\frac{\left(k_{\mathrm{T}}+k_{\mathrm{TET}} X_{0}\right)^{2}}{k_{2}}
$$

Equation 4 shows that this threshold will increase with QD concentration. The data for TIPS-Tc with $2 \mathrm{mg} / \mathrm{mL}$ of $\mathrm{PbS} /$ TetCAL QDs show a change from linear to square-root dependence at a threshold intensity of $2.9 \pm 1.0 \mathrm{~mW} / \mathrm{cm}^{2}$, consistent with the value of $2.0 \pm 1.2 \mathrm{~mW} / \mathrm{cm}^{2}$ expected from eq 4 and the nsTA kinetic parameters. In contrast, the solution with the higher concentration of QDs $(100 \mathrm{mg} / \mathrm{mL})$ shows little deviation from linearity over the range of intensities studied, indicating an insignificant effect from the bimolecular decay of triplets. The PLQE is therefore maintained up to solar-equivalent fluences (the photon flux available for absorption by TIPS-Tc under the AM1.5G spectrum) (Figure S30 and supplementary section 11). This shows that the TIPS$\mathrm{Tc}+\mathrm{PbS} / \mathrm{TetCAL}$ solutions are appropriate to use as a SF-PM under practical conditions.

Figure $4 \mathrm{~b}$ shows simulated normalized photon multiplication efficiencies, $\eta_{\mathrm{PM} /} \eta_{\mathrm{QD}}$, across a range of power flux and $\mathrm{PbS} /$ TetCAL QD concentrations. The results reveal that the intrinsic QD PLQE can be exceeded for a wide range of configurations. However, if we aim for a higher value of $\eta_{\mathrm{PM} /} \eta_{\mathrm{QD}}$ equal to $0.95 \eta_{\mathrm{SF}}$ (corresponding to $95 \%$ of the initial singlet fission efficiency) this significantly reduces the useful parameter space, as shown by the highlighted contour in Figure $4 \mathrm{~b}$, where the dotted red line corresponds to solar fluence. Thus, a minimum concentration of $30-40 \mathrm{mg} / \mathrm{mL}$ of QDs is required to efficiently harvest the bulk of the triplets generated at solar fluence for this system. As we have shown, this is limited by the bimolecular triplet-triplet recombination of TIPS-Tc.

\section{CONCLUSION}

We demonstrated a solution-based bulk SF-PM system in which $>95 \%$ of incident photons are absorbed by the singlet fission material, TIPS-Tc. Efficient singlet fission occurs in the solution phase before quantitative harvesting of the triplet excitons via a low concentration of $\mathrm{PbS}$ QDs $(\leq 50 \mathrm{mg} / \mathrm{mL})$ followed by emission of IR photons. We have shown that in order to obtain efficient harvesting of the fission generated triplets it is necessary to engineer the surface ligands on the $\mathrm{PbS}$ QDs. TetCAL ligands are shown to be much more efficient that either OA or HA. Several surprising results are uncovered; for instance, while the transport of the TIPS-Tc triplets to the $\mathrm{PbS} / \mathrm{TetCAL} \mathrm{QD}$ is the rate-limiting step, it occurs more slowly than would be expected for a purely diffusion-limited process, suggesting that there exists a mixed nature of the $\mathrm{QD}$ ligand coverage (consisting of both the TetCAL and OA). This leads to the need for multiple collisions before transfer is achieved to the TetCAL ligand. We also find that bimolecular recombination of triplets is the major loss channel and limits the photon multiplication performance at high fluences. However, it is possible to arrange a sufficiently high concentration of QDs $(30-50 \mathrm{mg} / \mathrm{mL})$ such that $95 \%$ of the triplets present can be harvested at solar fluence but still minimize parasitic absorption such that at energies below the absorption of TIPS-Tc less than 5\% of photons are absorbed by the PbS QDs. These results thus establish that it is possible to have a photon multiplication scheme that can function at solar fluence and shows the potential of singlet fission photon multiplication as a means to break the Shockley-Queisser limit. Currently, the limiting performance factors are the PLQE of the PbS/TetCAL quantum dots and the TIPS-Tc singlet fission yield. With the current singlet fission yield of $135 \%$, a QD with PLQE larger than $80 \%$ would be required in order to achieve an overall photon multiplication yield greater than $100 \%$.

Future improvements to the photon multiplication scheme should focus on increasing the transfer rate, resulting in a larger parameter space where triplets are effectively extracted with the need for lower concentration of QDs (Figure S29). A reduction in the rate of bimolecular recombination of triplets would also be highly desirable, for instance, by tuning the electronic structure such that TTA events lead solely to the 
reformation of the singlet state, which could then be recycled. Lessons can be learned from the photon upconversion field, where TTA yields for singlet generation can approach $100 \% .^{30}$ Ultimately, the SF-PM will require solid-state implementation, which will require careful control of nanomorphology, as well as energetics and surface chemistry of the QDs. The solutionbased SF-PM system we have established here serves as a convenient and highly tunable platform to understand the fundamental photophysics of the triplet transfer process from organic semiconductors to QDs and to test material combinations, energetics, and surface chemistries in order to guide the future development of solid-state SF-PM systems.

\section{ASSOCIATED CONTENT}

\section{S Supporting Information}

The Supporting Information is available free of charge on the ACS Publications website at DOI: 10.1021 /jacs.9b06584. Additional data related to this publication is available at the University of Cambridge data repository at https://doi.org/ 10.17863/CAM.42140.

Methods; absorption measurements; PLQE; kinetic modeling; diffusion-limited reactions; transient PL kinetic model; transient absorption; solar-equivalent fluence; steady-state modeling; steady-state IR PL; magnetic field-dependent PL; references (PDF)

\section{AUTHOR INFORMATION}

\section{Corresponding Author}

*ar525@cam.ac.uk

\section{ORCID $\odot$}

Jesse R. Allardice: 0000-0002-1969-7536

Arya Thampi: 0000-0001-6003-5991

Victor Gray: 0000-0001-6583-8654

Zhilong Zhang: 0000-0001-9903-4945

Neil C. Greenham: 0000-0002-2155-2432

John E. Anthony: 0000-0002-8972-1888

Akshay Rao: 0000-0003-4261-0766

\section{Notes}

The authors declare no competing financial interest.

\section{ACKNOWLEDGMENTS}

We thank the Winton Programme for the Physics of Sustainability and the Engineering and Physical Sciences Research Council for funding. This project has received funding from the European Research Council (ERC) under the European Union's Horizon 2020 research and innovation programme (grant agreement No 758826). J.R.A. acknowledges Cambridge Commonwealth European and International Trust for financial support. J.X. acknowledges EPSRC Cambridge NanoDTC, EP/L015978/1 for financial support. J.E.A. and A.J.P. acknowledge the U.S. National Science Foundation (DMREF-1627428) for support of organic semiconductor synthesis. V.G. acknowledges funding from the Swedish Research Council, Vetenskapsrådet 2018-00238.

\section{REFERENCES}

(1) Nelson, J. The physics of solar cells; World Scientific Publishing Co., 2003.

(2) NREL. Best Research-Cell Efficiencies; https://www.nrel.gov/ pv/assets/pdfs/best-research-cell-efficiencies-190416.pdf (Accessed May 15, 2019).
(3) Green, M. A.; Emery, K.; Hishikawa, Y.; Warta, W.; Dunlop, E. D. Solar cell efficiency tables (Version 45). Prog. Photovoltaics 2015, 23, 1-9.

(4) Shockley, W.; Queisser, H. J. Detailed balance limit of efficiency of $\mathrm{p}-\mathrm{n}$ junction solar cells. J. Appl. Phys. 1961, 32, 510-519.

(5) Smith, M. B.; Michl, J. Recent advances in singlet fission. Annu. Rev. Phys. Chem. 2013, 64, 361-86.

(6) Xia, J.; Sanders, S. N.; Cheng, W.; Low, J. Z.; Liu, J.; Campos, L. M.; Sun, T. Singlet Fission: Progress and Prospects in Solar Cells. Adv. Mater. 2017, 29, 1601652.

(7) Merrifield, R. E. (Triplet Annihilation, Simple) Theory of Magnetic Field Effects on the Mutual Annihilation of Triplet Excitons. J. Chem. Phys. 1968, 48, 4318.

(8) Merrifield, R. E.; Avakian, P.; Groff, R. P. Fission of singlet excitons into pairs of triplet excitons in tetracene crystals. Chem. Phys. Lett. 1969, 3, 386-388.

(9) Geacintov, N.; Pope, M.; Vogel, F. Effect of Magnetic Field on the Fluorescence of Tetracene Crystals: Exciton Fission. Phys. Rev. Lett. 1969, 22, 593-596.

(10) Pope, M.; Geacintov, N. E.; Vogel, F. Singlet Exciton Fission and Triplet-Triplet Exciton Fusion in Crystalline Tetracene. Mol. Cryst. 1969, 6, 83.

(11) Dexter, D. L. Two ideas on energy transfer phenomena: Ionpair effects involving the $\mathrm{OH}$ stretching mode, and sensitization of photovoltaic cells. J. Lumin. 1979, 18-19, 779-784.

(12) MacQueen, R. W.; et al. Crystalline silicon solar cells with tetracene interlayers: the path to silicon-singlet fission heterojunction devices. Mater. Horiz. 2018, 5, 1065-1075.

(13) Ehrler, B.; Wilson, M. W. B.; Rao, A.; Friend, R. H.; Greenham, N. C. Singlet exciton fission-sensitized infrared quantum dot solar cells. Nano Lett. 2012, 12, 1053-1057.

(14) Congreve, D. N.; et al. External quantum efficiency above $100 \%$ in a singlet-exciton-fission-based organic photovoltaic cell. Science 2013, 340, 334-7.

(15) Pazos-Outon, L. M.; Lee, J. M.; Futscher, M. H.; Kirch, A.; Tabachnyk, M.; Friend, R. H.; Ehrler, B. A Silicon-Singlet Fission Tandem Solar Cell Exceeding 100\% External Quantum Efficiency with High Spectral Stability. ACS Energy Lett. 2017, 2, 476.

(16) Rao, A.; Friend, R. H. Harnessing singlet exciton fission to break the Shockley-Queisser limit. Nat. Rev. Mater. 2017, 2, 1-12.

(17) Futscher, M. H.; Rao, A.; Ehrler, B. The Potential of Singlet Fission Photon Multipliers as an Alternative to Silicon-Based Tandem Solar Cells. ACS Energy Lett. 2018, 3, 2587.

(18) Thompson, N. J.; et al. Energy harvesting of non-emissive triplet excitons in tetracene by emissive $\mathrm{PbS}$ nanocrystals. Nat. Mater. 2014, 13, 1039-1043.

(19) Tabachnyk, M.; et al. Resonant energy transfer of triplet excitons from pentacene to PbSe nanocrystals. Nat. Mater. 2014, 13, $1033-1038$.

(20) Huang, Z.; et al. Hybrid Molecule - Nanocrystal Photon Upconversion Across the Visible and Near-Infrared. Nano Lett. 2015, $15,5552-5557$.

(21) Mongin, C.; Garakyaraghi, S.; Razgoniaeva, N.; Zamkov, M.; Castellano, F. N. Direct observation of triplet energy transfer from semiconductor nanocrystals. Science (Washington, DC, U. S.) 2016, $351,369-372$.

(22) Mahboub, M.; Maghsoudiganjeh, H.; Pham, A. M.; Huang, Z.; Tang, M. L. Triplet Energy Transfer from $\mathrm{PbS}(\mathrm{Se})$ Nanocrystals to Rubrene: the Relationship between the Upconversion Quantum Yield and Size. Adv. Funct. Mater. 2016, 26, 6091-6097.

(23) Huang, Z.; Tang, M. L. Designing Transmitter Ligands That Mediate Energy Transfer between Semiconductor Nanocrystals and Molecules. J. Am. Chem. Soc. 2017, 139, 9412-9418.

(24) Nishimura, N.; Allardice, J. R.; Xiao, J.; Gu, Q.; Gray, V.; Rao, A.; et al. Photon upconversion utilizing energy beyond the band gap of crystalline silicon with a hybrid TES-ADT/PbS quantum dots system. Chem. Sci. 2019, 10, 4750-4960. 
(25) Nienhaus, L.; et al. Speed Limit for Triplet-Exciton Transfer in Solid-State PbS Nanocrystal-Sensitized Photon Upconversion. ACS Nano 2017, 11, 7848-7857.

(26) Li, X.; Huang, Z.; Zavala, R.; Tang, M. L. Distance-Dependent Triplet Energy Transfer between CdSe Nanocrystals and Surface Bound Anthracene. J. Phys. Chem. Lett. 2016, 7, 1955-1959.

(27) Stern, H. L.; et al. Identification of a triplet pair intermediate in singlet exciton fission in solution. Proc. Natl. Acad. Sci. U. S. A. 2015, 112, 7656-7661.

(28) Davis, N. J. L. K.; et al. Singlet Fission and Triplet Transfer to PbS Quantum Dots in TIPS-Tetracene Carboxylic Acid Ligands. J. Phys. Chem. Lett. 2018, 9, 1454-1460.

(29) Dover, C. B.; et al. Endothermic singlet fission is hindered by excimer formation. Nat. Chem. 2018, 10, 305-310.

(30) Cheng, Y. Y.; et al. Kinetic Analysis of Photochemical Upconversion by Triplet-Triplet Annihilation: Beyond Any Spin Statistical Limit. J. Phys. Chem. Lett. 2010, 1, 1795-1799.

(31) Huang, Z.; et al. PbS/CdS Core-Shell Quantum Dots Suppress Charge Transfer and Enhance Triplet Transfer. Angew. Chem., Int. Ed. 2017, 56, 16583-16587.

(32) Shoup, D.; Lipari, G.; Szabo, A. Diffusion-controlled bimolecular reaction rates. The effect of rotational diffusion and orientation constraints. Biophys. J. 1981, 36, 697-714. 